\title{
Una interpretación física de la Constante de Planck
}

\author{
*WILSON P. ALVAREZ-SAMANIEGO', BORYS ALVAREZ-SAMANIEGO', DOUGLAS MOYA-ALVAREZ \\ 'Núcleo de Investigadores Científicos \\ Facultad de Ingeniería, Ciencias Físicas y Matemática \\ Universidad Central del Ecuador (UCE), Quito, Ecuador \\ *Correspondencia: balvarez@uce.edu.ec \\ Recibido: 27 de mayo de 2013, Aceptado: 15 de octubre de 2013
}

\section{Resumen}

De acuerdo a la interpretacion comunmente aceptada de la Mecánica Cuántica solo se puede hablar de la existencia de las partículas elementales cuando ellas son detectadas en un experimento o con un aparato clásico de medida.

Esto ha conllevado a tergiversaciones respecto de la existencia objetiva de la realidad, puesto que se requeriría un observador para que se materialice la partícula elemental. Este problema se resuelve cuando se conceptúa a la partícula elemental en interacción permanente con el resto del Universo de modo que así su existencia no dependerá de ningún observador inteligente y se rescatará el rango objetivo de las partculas elementales. En este artículo se estudia la interacción entre las partículas elementales y un campo de acción de fondo de carácter estocástico y se determina que la desviación estándar de la interacción partícula-campo de fondo es esencialmente la constante de Planck, pues aparecen de manera natural las expresiones de la energía según el Postulado de Planck y de la cantidad de movimiento de acuerdo a la tesis de Lovis de Broglie. Además, se obtiene de forma natural la expresión de la función de onda de una partícula libre a partir de la resolución de la ecuación integral que liga la cantidad de movimiento y la energía con el vector de onda y la frecuencia angular de una distribución gaussiana. También, se deduce la ecuación de Schrödinger como consecuencia de la expresión de la función de onda de una partícula elemental en un campo potencial. Finalmente, se obtiene el Principio de Incertidumbre. La teoría presentada en este artículo rescata el carácter objetivo de la Mecánica Cuántica por el cual las partículas elementales existen independientemente de los observadores ya que el aparato de medida clásico en esta teoría, introducida por los autores, es el propio Universo.

Palabras clave: Campo de acción de fondo, constante de Planck, desviación estándar, densidad de distribución de la acción.

\section{A physical interpretation of the Planck Constant}

Abstract

According to the commonly accepted interpretation of Quantum Mechanics, it is only possible to talk about the existence of elementary particles when they are detected by an experiment or by a classical measurement device. This has led to distortions with regard to the objective existence of reality, since an observer would be necessary to materialize the elementary particle. This issue is solved when the elementary particle is placed in permanent interaction with the rest of the universe, so its existence would not depend on any intelligent observer and thus the objective range of elementary particles would be recovered. This article explores the interaction between elementary particles and a background action eld of stochastic character and it is also determined that the standard deviation of the particle-background field interaction is essentially the Planck constant, since the 
expressions of the energy according to the Planck postulate and the momentum according to the Louis de Broglie thesis follow in a natural way. In addition, the expression for the wave function of a free particle is obtained from the solution of the integral equation that relates the momentum and the energy with the wave vector and the angular frequency of a Gaussian distribution. Also, the Schrödinger equation is deduced from the expression for the wave function of an elementary particle in a potential field. Finally, the uncertainty principle is obtained.

The theory presented in this article rescues the objective nature of Quantum Mechanics which implies that elementary particles exist independently of observers since the classical measurement device in this theory, introduced by the authors, is the universe itself.

Key words: Background action field, Planck's constant, standard deviation, action density distribution.

\section{Introducción}

Sir Isaac Newton (1642-1727) cuando concibió el principio de inercia imaginó la existencia de un objeto masivo que mantenía su estado en movimiento rectilíneo uniforme. De hecho, Newton suponía que los objetos físicos pueden existir por sí mismos, de manera que podemos abstraer su existencia del resto del Universo sin alterar las cualidades dinámicas del mencionado objeto. Este punto de vista concordaba con la Filosofía Racionalista de René Descartes (1596-1650) en la que se plantea por primera vez en la historia de Occidente la existencia de un ser humano independiente del entorno social y, por lo tanto, la afirmación del individuo en sí y de por sí, el cual es uno de los principios fundamentales sobre los que se levantaría la futura ideología burguesa. Este elemento ideológico penetró en la Ciencia haciendo que esta solamente sea exitosa en el mundo macroscópico, donde el experimentador puede controlar las operaciones de medida y sus errores haciéndolos tan pequeños como él desee, lo que equivale a considerar el límite clásico de la constante de Planck igual a cero.

Sin embargo, en el mundo microscópico donde existen objetos tan pequeños como son las moléculas, átomos, partículas elementales, cuyas dimensiones tienen órdenes menores o iguales a $10^{-10} \mathrm{~m}$, esas partículas están inmersas en un océano de interacciones cuyo origen está en la propia existencia de cada una de ellas extendidas a todo el Universo. Este océano de interacciones fluctúa estocásticamente haciendo imposible determinar simultáneamente con precisión la posición y velocidad de las partículas elementales. En este trabajo se demuestra que la función de densidad de la acción a la que está sometida la partícula es una gaussiana, lo cual es una consecuencia del Teorema Central del Límite. Expresando esta función en el dominio de la frecuencia se encuentra otra distribución gaussiana cuyo exponente es, nuevamente, una función cuadrática de una combinación lineal de las variables espacial y temporal, y donde los coeficientes de la variable espacial y de la variable temporal en el exponente son el módulo del vector de onda $k$ y la frecuencia angular $\omega=\omega(\vec{k})$ de la onda respectivamente. De ahí se deduce que el módulo de la cantidad de movimiento $P$ es proporcional a la desviación estándar $\sigma$ del campo de acción de fondo por el módulo del vector de onda, y la energía $E$ de la partícula es también proporcional a la frecuencia angular por la desviación estándar del campo de acción de fondo.

Se demuestra además que el núcleo de la ecuación integral que permite transformar la representación en el dominio de la frecuencia a la representación en el dominio del espacio-tiempo comunes es el producto de dos deltas de Dirac cuyos argumentos son $\frac{P}{\bar{h}}-k$ y $\frac{E}{\bar{h}}-\omega$ respectivamente. Expresando estas dos distribuciones mediante una superposición continua de ondas planas se concluye que la partícula puede ser representada por una función compleja de la forma

$$
\psi(x, t)=\frac{1}{\sqrt{2 \pi}} e^{i\left(\frac{P x-E t}{\bar{h}}\right)} .
$$

A partir de la última expresión y usando la relación no relativista de la energía y el hecho de que la función 
de onda del electrón en un potencial, $U(\vec{r})$, se puede expresar como una superposición de ondas planas, se procede a deducir en la Sección 3 la ecuación de Schrödinger de la partícula elemental.

En la Sección 4 se demuestra el Principio de Incertidumbre desde la función de densidad de probabilidad de la acción del campo de fondo de una partícula libre. Vale mencionar que este principio también se puede obtener como una consecuencia matemática del Teorema de Parseval [3]. Además, se obtiene la constante de proporcionalidad entre la desviación estándar y la constante de Planck para la Mecánica Cuántica tradicional. Finalmente, se encuentra la ecuación correspondiente a la energía del punto cero del sistema partícula libre-campo de acción de fondo (ver ecuación (4.2)).

\section{Función de distribución de la acción e interpretación física de la Constante de Planck}

Vamos a comenzar deduciendo la densidad de distribución gaussiana de la acción del campo de fondo sobre la partícula. Para ello, se inicia obteniendo la función de densidad que se presenta en un conjunto repetido de mediciones. De esta forma, se determina el valor real de la medición como el promedio de los valores experimentales obtenidos y se consigue, además, la dispersión estadística de datos de este proceso.

A continuación, se enuncian los supuestos para la obtención de la densidad de distribución gaussiana:

1. Pequeños errores son más probables que grandes errores.

2. Para un valor real $p$ dado, las dispersiones estadísticas en $\pm \varepsilon$, tienen igual probabilidad.

3. En la presencia de varias observaciones sobre la misma cantidad, el valor más probable de esa cantidad es el promedio de las observaciones.

Carl Friedrich Gauss (1777-1855) se refirió a dicho proceso como "el problema más importante de las Matemáticas en la Filosofía Natural".

Ahora, se procede a la deducción de la densidad de distribución gaussiana asociada a la acción de la par- tícula. Sea $p \in \mathbb{R}$ el verdadero valor (pero desconocido) de la medida de la cantidad física. Se efectúan $n \in \mathbb{N}$ observaciones independientes del experimento asociado a la medida de la cantidad física $p$, dichas observaciones dan como resultado las medidas $M_{1}, M_{2}, \ldots, M_{n}$. Sea además $\Phi$ la función de densidad de probabilidad del error aleatorio. Se supone que la función $\Phi$ es diferenciable, y que $\Phi(x) \neq 0$, para todo $x \in \mathbb{R}$.

La suposición 1 anterior implica que $\Phi$ tiene un valor máximo en $x=0$, mientras que la afirmación 2 implica que $\Phi(x)=\Phi(-x)$, para todo $x \in \mathbb{R}$.

Se define la función $f: \mathbb{R} \rightarrow \mathbb{R}$ por

$$
f(x):=\frac{\phi^{\prime}(x)}{\phi(x)}, \text { para todo } x \in \mathbb{R} .
$$

Entonces,

$$
f(-x)=-f(x), \text { para todo } x \in \mathbb{R} .
$$

Note que $X_{i}:=M_{i}-p$ denota la variable aleatoria asociada al error de la i-ésima medida. Ya que estas medidas (y errores) se asumen estocásticamente independientes, se sigue que

$$
\begin{aligned}
\Omega_{n} & :=\phi\left(M_{1}-p\right) \ldots \phi\left(M_{n}-p\right) \\
& =\prod_{i=1}^{n} \phi\left(M_{i}-p\right)
\end{aligned}
$$

es la densidad conjunta asociada a los $n$ errores. Por otro lado, de la afirmación 3 se tiene que

$$
\bar{M}_{n}:=\frac{M_{1}+M_{2}+\ldots .+M_{n}}{n}
$$

es el estimador verosímil de $p$. En otras palabras, dadas las medidas $M_{1}, M_{2}, \ldots, M_{n^{\prime}}$ al escoger $p=\bar{M}_{n}$, se maximiza el valor de $\Omega_{n}$.

A continuación, se evalúa el valor de la derivada de $\Omega_{n}$ en el punto $p=\bar{M}_{n}$.

$$
\begin{aligned}
0 & =\left.\frac{d \Omega_{n}}{d p}\right|_{p=\bar{M}_{n}} \\
& =-\sum_{i=1}^{n} \phi^{\prime}\left(M_{i}-\bar{M}_{n}\right) \prod_{j \neq i} \phi\left(M_{j}-\bar{M}_{n}\right) \\
& =-\sum_{i=1}^{n} \frac{\phi^{\prime}\left(M_{i}-\bar{M}_{n}\right)}{\phi\left(M_{i}-\bar{M}_{n}\right)} \prod_{k=1}^{n} \phi\left(M_{k}-\bar{M}_{n}\right)
\end{aligned}
$$




$$
\begin{aligned}
& =-\sum_{i=1}^{n} f\left(M_{i}-\bar{M}_{n}\right) \Omega_{n} \\
& =-\Omega_{n} \sum_{i=1}^{n} f\left(M_{i}-\bar{M}_{n}\right) .
\end{aligned}
$$

Entonces,

$$
\sum_{i=1}^{n} f\left(M_{i}-\bar{M}_{n}\right)=0
$$

Se consideran ahora $M$ y $N$ dos variables aleatorias arbitrarias que podrían representar diversas magnitudes físicas tales como longitud, energía u otras. En vista que las medidas dadas por las variables aleatorias $M_{i}, i=1, \ldots, n$, pueden tomar valores arbitrarios, se toma a continuación

$$
\begin{aligned}
& M_{1}=M, \\
& M_{2}=M_{3}=\cdots=M_{n}=M-n N
\end{aligned}
$$

Para tal conjunto de medidas, se obtiene por lo tanto que

$$
\bar{M}_{n}=M-(n-1) N .
$$

Luego, de la ecuación (2.1) y considerando (2.2), se tiene que

$$
\begin{aligned}
& f(M-[M-(n-1) N])+ \\
(n-1) f(M-n N-(M-(n-1) N)) & \\
= & 0 .
\end{aligned}
$$

Por lo tanto,

$$
f[(n-1) N]=(n-1) f(N) .
$$

De la paridad y de la continuidad de $f$, se sigue que existe $k \in \mathbb{R}$ tal que $f(x)=k x$, para todo $x \in \mathbb{R}$. Luego,

$$
f(\lambda x)=\lambda k x=\lambda f(x), \text { para todo } x \in \mathbb{R}
$$

Entonces,

$$
\frac{\phi^{\prime}(x)}{\phi(x)}=k x, \quad \text { para todo } x \in \mathbb{R} .
$$

De donde se sigue que

$$
\phi(x)=A e^{\frac{k x^{2}}{2}}, \text { para todo } x \in \mathbb{R} .
$$

Definamos ahora,

$$
k=-\frac{1}{\sigma^{2}}
$$

Así,

$$
\phi(x)=A e^{-\frac{x^{2}}{2 \sigma^{2}}}, \text { para todo } x \in \mathbb{R}
$$

De la expresión anteriory suponiendo que $\int_{\mathbb{R}} \phi(x) d x=1$, se observa que la constante $A$ está dada por

$$
A=\frac{1}{\sqrt{2 \pi} \sigma} .
$$

En consecuencia,

$$
\phi(x)=\frac{1}{\sqrt{2 \pi} \sigma} e^{-\frac{x^{2}}{2 \sigma^{2}}}, \text { para todo } x \in \mathbb{R}
$$

Luego, la función de densidad de distribución de la acción del campo de fondo viene dada por:

$$
\phi(S)=\frac{1}{\sqrt{2 \pi} \sigma} e^{-\frac{S^{2}}{2 \sigma^{2}}},
$$

donde $S=P x$-Et, aquí $P$ corresponde al momentum de la partícula y $E$ denota su energía.

A continuación, se presenta una interpretación de la desviación estándar $\sigma$ en función de la constante de Planck $\bar{h}$ (ver Observación 2.1 ) abajo. Usando una ecuación integral para representar al término $\sqrt{2 \pi} \sigma \phi(S)$, se puede escribir

$$
\begin{aligned}
& \exp \left(-\frac{S^{2}}{2 \sigma^{2}}\right) \\
& =\iint_{\mathbb{R}^{2}} B(P, E, k, \omega) C(k x-\omega t) d k d \omega,
\end{aligned}
$$

donde en el lado izquierdo de la última ecuación aparece esencialmente la función de densidad de distribución de la acción $S$ en el espacio-tiempo, $C(k x-\omega t)$ corresponde a la representación de esa distribución en el espacio de Fourier y $B(P, E, k, \omega)$ corresponde al Jacobiano de la transformación entre esos dos espacios. Vale mencionar que este tratamiento fue introducido por $A$. Einstein en uno de sus tres famosos artícu- 
los publicados en 1905 para describir el movimiento Browniano y se lo considera como el inicio de la teoría de Procesos Estocásticos. Sea

$$
\theta:=k x-\omega t
$$

Derivando (2.4) respecto a $x$, y usando formalmente el Teorema de Convergencia Dominada (Teorema 6.1) con las hipótesis de regularidad e integrabilidad apropiadas, se obtiene

$$
\begin{aligned}
& -\frac{S}{\sigma^{2}} \frac{\partial S}{\partial x} \exp \left(-\frac{S^{2}}{2 \sigma^{2}}\right)= \\
& \iint_{\mathbb{R}^{2}} B(P, E, k, \omega) C^{\prime}(k x-\omega t) \frac{\partial \theta}{\partial x} d k d \omega .
\end{aligned}
$$

Derivando ahora (2.4) respecto a la varible $t$, y usando formalmente el Teorema de Convergencia Dominada, se ve que

$$
\begin{aligned}
& -\frac{S}{\sigma^{2}} \frac{\partial S}{\partial t} \exp \left(-\frac{S^{2}}{2 \sigma^{2}}\right)= \\
& \iint_{\mathbb{R}^{2}} B(P, E, k, \omega) C^{\prime}(k x-\omega t) \frac{\partial \theta}{\partial t} d k d \omega .
\end{aligned}
$$

Luego,

$$
\begin{aligned}
& -\frac{S}{\sigma^{2}} \frac{\partial S}{\partial x} \int_{\mathbb{R}^{2}} B(P, E, k, \omega) C(\theta) d k d \omega \\
& =\iint_{\mathbb{R}^{2}} B(P, E, k, \omega) C^{\prime}(\theta) \frac{\partial \theta}{\partial x} d k d \omega, \\
& -\frac{S}{\sigma^{2}} \frac{\partial S}{\partial t} \iint_{\mathbb{R}^{2}} B(P, E, k, \omega) C(\theta) d k d \omega \\
& =\iint_{\mathbb{R}^{2}} B(P, E, k, \omega) C^{\prime}(\theta) \frac{\partial \theta}{\partial t} d k d \omega .
\end{aligned}
$$

Así,

$$
\begin{aligned}
& \iint_{\mathbb{R}^{2}} B(P, E, k, \omega) \\
& {\left[\frac{S}{\sigma^{2}} \frac{\partial S}{\partial x} C(\theta)+C^{\prime}(\theta) \frac{\partial \theta}{\partial x}\right] d k d \omega=0,} \\
& \iint_{\mathbb{R}^{2}} B(P, E, k, \omega) \\
& {\left[\frac{S}{\sigma^{2}} \frac{\partial S}{\partial t} C(\theta)+C^{\prime}(\theta) \frac{\partial \theta}{\partial t}\right] d k d \omega=0 .}
\end{aligned}
$$

Ahora, se considera

$$
\frac{S}{\sigma^{2}} \frac{\partial S}{\partial x} C(\theta)+C^{\prime}(\theta) \frac{\partial \theta}{\partial x}=0,
$$

y

$$
\frac{S}{\sigma^{2}} \frac{\partial S}{\partial t} C(\theta)+C^{\prime}(\theta) \frac{\partial \theta}{\partial t}=0 .
$$

Multiplicando la ecuación (2.5) por $x$ y la ecuación (2.6) por $d t$ y sumando, se consigue

$$
\begin{aligned}
& {\left[C^{\prime}(\theta) \frac{\partial \theta}{\partial x} d x+C^{\prime}(\theta) \frac{\partial \theta}{\partial t} d t\right.} \\
& \left.+C(\theta)\left(\frac{S}{\sigma^{2}} \frac{\partial S}{\partial x} d x+\frac{S}{\sigma^{2}} \frac{\partial S}{\partial t} d t\right)\right]=0 .
\end{aligned}
$$

Luego,

$$
d C(\theta)+C(\theta) d\left(\frac{S^{2}}{2 \sigma^{2}}\right)=0 .
$$

Entonces,

$$
\frac{d C(\theta)}{C(\theta)}+d\left(\frac{S^{2}}{2 \sigma^{2}}\right)=0 .
$$

De donde, integrando se obtiene que

$$
\ln (C(\theta))+\frac{S^{2}}{2 \sigma^{2}}=\alpha,
$$

donde $\alpha$ es una constante. Luego,

$$
C(\theta)=e^{\alpha} e^{-\frac{s^{2}}{2 \sigma^{2}}} .
$$

Así, se tiene que

$$
\begin{gathered}
C(k x-\omega t)=e^{\alpha} e^{-\frac{(P x-E t)^{2}}{2 \sigma^{2}}}, \\
\text { para todo } x \in \mathbb{R}, t \in R .
\end{gathered}
$$

Tomando $t=0$ en la ecuación (2.7), se sigue que

$$
C(y)=e^{\alpha} e^{-\frac{1}{2}\left(\frac{P}{\sigma k}\right)^{2} y^{2}}, \text { para todo } y \in \mathbb{R} .
$$

Por otro lado, escogiendo $x=0$ en (2.7), se ve que

$$
C(y)=e^{\alpha} e^{-\frac{1}{2}\left(\frac{E}{\sigma \omega}\right)^{2} y^{2}} \text {, para todo } y \in \mathbb{R} \text {. }
$$

De las dos expresiones anteriores se obtiene la siguiente relación: 


$$
\frac{P}{k}=\frac{E}{\omega}
$$

Observación 2.1. Sin embargo, se conoce de la Mecánica Cuántica habitual que $\frac{P}{k}=\frac{E}{\omega}=\bar{h}$. Además, de (2.7) se sigue que $\frac{P}{\sigma}$ es proporcional a $k$, de lo cual se tiene que $\sigma$ es proporcional a $\bar{h}$ (existe $\beta>0$ tal que $\bar{h}=\beta \sigma)$. De aquí en adelante, sin pérdida de generalidad, se escoge $\alpha=0$ en (2.7).

Finalmente, se procede a construir la función de onda planar asociada a la partícula libre. Usando la ecuación (2.4) y la constante $\beta>0$ mencionada en la Observación 2.1 , se tiene que

$$
\begin{aligned}
e^{-\frac{(P x-E t)^{2}}{2 \sigma^{2}}} & \int_{\mathbb{R}^{2}} B(P, E, k, \omega) C(k x-\omega t) d k d \omega \\
= & \iint_{\mathbb{R}^{2}} B(P, E, k, \omega) e^{-\frac{\beta^{2}(k x-\omega t)^{2}}{2}} d k d w .
\end{aligned}
$$

Tomando por ejemplo $B(P, E, k, \omega)=\delta\left(\frac{P}{\beta \sigma}-k\right) \delta\left(\frac{E}{\beta \sigma}-\omega\right)$ se ve que

$$
\begin{aligned}
& e^{-\frac{\left(P_{x}-E t\right)^{2}}{2 \sigma^{2}}}= \\
& \iint_{\mathbb{R}^{2}} \delta\left(\frac{P}{\beta \sigma}-k\right) \delta\left(\frac{E}{\beta \sigma}-\omega\right) \\
& \times e^{-\frac{\beta^{2}(k x-\omega t)^{2}}{2}} d k d \omega .
\end{aligned}
$$

Usando la transformada de Fourier $F$, también denotada por ${ }^{\wedge}$, se tiene que

$$
\frac{\widehat{e^{i a}}}{\sqrt{2 \pi}}=\delta_{a}, \quad \text { para todo } a \in \mathbb{R}
$$

donde $\delta_{a^{\prime}}$ para $a \in \mathbb{R}$, representa la distribución temperada definida por

$$
\delta_{a}(\varphi)=\varphi(a), \quad \text { para } \phi \in S(\mathbb{R}),
$$

aquí $S(\mathbb{R})$ representa el espacio de Schwartz de las funciones rápidamente decrecientes en $\mathbb{R}$. Luego, se sigue (usando la notación usual en Física) que y

$$
\delta\left(\frac{E}{\bar{h}}-\omega\right)=\frac{1}{\sqrt{2 \pi}} \int_{-\infty}^{+\infty} e^{-i\left(\frac{E}{\bar{h}}-\omega\right) t} d t .
$$

Entonces, siguiendo con la notación usual en Mecánica Cuántica, se ve que

$$
\begin{aligned}
& \delta\left(\frac{P}{\bar{h}}-k\right) \delta\left(\frac{E}{\bar{h}}-\omega\right) \\
& =\frac{1}{2 \pi} \iint_{\mathbb{R}^{2}} e^{i \frac{(P x-E t)}{\bar{h}}} e^{-i(k x-\omega t)} d x d t .
\end{aligned}
$$

De donde, usando la condición de normalización de Dirac, se observa la representación espacial de la función de onda de la partícula libre dada por

$$
\psi(x, t)=\frac{1}{\sqrt{2 \pi}} e^{i\left(\frac{P x-E t}{\bar{h}}\right)} .
$$

\section{Deducción de la ecuación de Schrödinger}

Si un electrón está sometido a una energía potencial, $U(\vec{r})$, éste forma el paquete de ondas

$$
\psi(\vec{r}, t)=\int D(\vec{k}) e^{i(\vec{k} \cdot \vec{r}-\omega(\vec{k}) t)} d^{3} \vec{k},
$$

donde $D$ es la representación en el espacio de momentums de la función de onda. Derivando respecto al tiempo la expresión anterior y usando formalmente el Teorema de Convergencia Dominada con las hipótesis convenientes, se obtiene que

$$
\begin{aligned}
& \frac{\partial \psi}{\partial t}(\vec{r}, t) \\
& =\int-i \omega(\vec{k}) D(\vec{k}) e^{i(k \cdot r-\omega(\vec{k}) t)} d^{3} \vec{k}
\end{aligned}
$$

y como $E=\bar{h} \omega(\vec{k})$, se ve que

$$
\frac{\partial \psi}{\partial t}(r, t)=\int-i \frac{E}{\bar{h}} D(\vec{k}) e^{i(\vec{k} \cdot r-\omega(\vec{k}) t)} d^{3} \vec{k}
$$

Luego,

$$
i \bar{h} \frac{\partial \psi}{\partial t}(r, t)=\int E D(\vec{k}) e^{i(\vec{k} \cdot r-\omega(\bar{k}) t)} d^{3} \vec{k}
$$

En el caso no relativista se conoce que

$$
E=\frac{P^{2}}{2 m}+U(\vec{r}) .
$$

$$
\delta\left(\frac{P}{\bar{h}}-k\right)=\frac{1}{\sqrt{2 \pi}} \int_{-\infty}^{+\infty} e^{i\left(\frac{P}{h}-k\right) x} d x
$$


Así que

$$
\begin{aligned}
& i \bar{h} \frac{\partial \psi}{\partial t}(\vec{r}, t) \\
& =\int_{C} \frac{P^{2}}{2 m} D(\vec{k}) e^{i(\vec{k} \cdot r-\omega(\vec{k}) t)} d^{3} \vec{k} \\
& +U(\vec{r}) \psi(\vec{r}, t) .
\end{aligned}
$$

Nuevamente, usando formalmente el Teorema de Convergencia Dominada con las hipótesis oportunas, se consigue

$$
\begin{aligned}
& -\nabla^{2} \psi(r, t) \\
& =\int k^{2} D(\vec{k}) e^{i(\vec{k} \cdot r-\omega(\vec{k}) t)} d^{3} \vec{k} \\
& =\int \frac{P^{2}}{\bar{h}^{2}} D(\vec{k}) e^{i(\vec{k} \cdot r-\omega(\vec{k}) t)} d^{3} \vec{k} .
\end{aligned}
$$

De donde se obtiene que

$$
\begin{aligned}
& -\frac{\bar{h}^{2}}{2 m} \nabla^{2} \psi(r, t) \\
& =\int \frac{P^{2}}{2 m} D(\vec{k}) e^{i(\vec{k} \cdot r-\omega(\vec{k}) t)} d^{3} \vec{k} .
\end{aligned}
$$

En consecuencia

$$
\begin{aligned}
& i \bar{h} \frac{\partial \psi}{\partial t}(r, t) \\
& =-\frac{\bar{h}^{2}}{2 m} \nabla^{2} \psi(\vec{r}, t)+U(\vec{r}) \psi(\vec{r}, t),
\end{aligned}
$$

que es la ecuación de Schrödinger.

\section{Deducción del Principio de Incertidumbre}

Consideramos una partícula libre cuya función de densidad de probabilidad de la acción del campo de fondo viene dada por

$$
\phi(S)=\frac{1}{\sqrt{2 \pi} \sigma} e^{-\frac{S^{2}}{2 \sigma^{2}}},
$$

donde

$$
S=\int_{0}^{t} L\left(t^{\prime}\right) d t^{\prime}
$$

corresponde a la acción de la partícula, $L=T-U$ es el Lagrangiano en la perspectiva de la Mecánica Clásica no relativista, $T=\frac{P^{2}}{2 m}$ representa la energía cinética de la partícula y $U$ es la energía potencial que en el caso de la partícula libre puede ser tomada como cero. Luego, la acción para la partícula libre viene dada por

$$
S=\int_{0}^{t} \frac{P^{2}}{2 m} d t^{\prime}=\frac{P^{2}}{2 m} t=\frac{P^{2}}{2} \frac{x}{m v}=\frac{P x}{2} .
$$

Por otro lado, la varianza de la acción está expresada por

$$
\sigma^{2}=\overline{S^{2}}-\bar{S}^{2},
$$

donde

$$
\overline{S^{2}}=\frac{1}{\sqrt{2 \pi} \sigma} \int_{\mathbb{R}} e^{-\frac{S^{2}}{2 \sigma^{2}}} S^{2} d S
$$

y

$$
\bar{S}=\frac{1}{\sqrt{2 \pi} \sigma} \int_{\mathbb{R}} e^{-\frac{S^{2}}{2 \sigma^{2}}} S d S=0 .
$$

Entonces,

$$
\sigma^{2}=\overline{S^{2}}=\overline{\left(\frac{P x}{2}\right)^{2}}
$$

Por lo tanto,

$$
\sigma=\frac{1}{2} \sqrt{\overline{(P x)^{2}}} .
$$

Como $\sigma$ representa la desviación estándar de la acción del campo de fondo de la partícula, entonces cualquier rectángulo en el espacio de fase de lados $\Delta x, \Delta P$ posee un área mayor o igual que $\sigma$, es decir

$$
\Delta x \Delta P \geq \sigma .
$$

Para coincidir con la Mecánica Cuántica usual se toma $\sqrt{(P x)^{2}}=\bar{h}$. Entonces, se tiene que

$$
\Delta x \Delta P \geq \sigma=\frac{\bar{h}}{2} .
$$

Se considera ahora el caso de menor incertidumbre, $\Delta x \Delta P=\sigma=\frac{\hbar}{2}$. Sea $\varphi$ la función de onda de la partícula libre correspondiente al caso de incertidumbre mínima en la representación de coordenadas. De la relación

$$
\phi(S) d S=|\varphi(x)|^{2} d x,
$$

la cual determina la probabilidad de que la partícula libre tenga una acción comprendida entre $S$ y $S+d S$ 
y que también es igual a la probabilidad de que la partícula libre en incertidumbre mínima esté entre $x y$ $x+d x$, se concluye que

$$
\varphi(x)=\left(\frac{P}{\bar{h} \sqrt{2 \pi}}\right)^{\frac{1}{2}} e^{-\frac{P^{2} x^{2}}{4 h^{2}}} .
$$

Substituyendo la última expresión en la ecuación de Schrödinger se obtiene finalmente que

$$
E=\frac{\bar{h} \omega}{2}=m c^{2},
$$

donde $E$ y $m$ son la energía y masa de la partícula libre respectivamente, $c$ es la velocidad de la luz y $\omega \equiv \frac{P^{2}}{2 \hbar m}$ representa la frecuencia natural de oscilación de la partícula en el interior del campo de acción de fondo. Este último resultado será analizado con más detalle en un próximo trabajo.

\section{Conclusiones}

A continuación algunas consecuencias de lo expuesto en las secciones anteriores.

- Se rescata el carácter objetivo de las partículas elementales, puesto que el aparato macroscópico de medida constituye el resto del Universo.

- Se recupera la naturaleza causal de la Teoría Cuántica, ya que las transiciones "espontáneas" son producto de las perturbaciones del campo de fondo que actúa sobre una partícula en estado cuántico "excitado" volviendo al estado estacionario de menor energía.

- De la Sección 2 se deduce que la constante de Planck es "esencialmente" la desviación estándar del campo de interacciones de fondo con la partícula.
- De la ecuación (2.8) y de la Observación 2.1 se deducen los Postulados de Planck y de-Broglie, estableciendo el carácter de la dualidad onda-corpúsculo de la Mecánica Cuántica tradicional.

- En la Sección 3 se deduce la ecuación de Schrödinger asociada a una partícula en un potencial, $U(\vec{r})$, la cual viene dada por la ecuación (3.1). Esta ecuación se obtuvo suponiendo que la función de onda de dicha partícula es una superposición de infinitas ondas planas. Por otro lado, en la Mecánica Cuántica tradicional la ecuación de Schrödinger se la postula como su principio dinámico (ver [4] para más detalles).

- En la Sección 4 se deduce, desde la teoría presentada en este artículo, el Principio de Incertidumbre. Además, se observa que para el caso de la Mecánica Cuántica habitual, la constante de proporcionalidad mencionada en la Observación 2.1 es $\beta=2$. Finalmente, se analiza la conducta física de una partícula libre en condiciones de incertidumbre mínima, obteniendo la ecuación (4.2) que corresponde a la energía del punto cero del sistema partícula-campo de acción de fondo.

\section{Apéndice}

TEOREMA 6.1 (Teorema de Convergencia Dominada de Lebesgue [1]). Sea $(\Omega, A, \mu)$ un espacio de medida. Sea $\left(f_{n}\right)_{n \in N}$ una sucesión de funciones integrables $\left(f_{n} \in L(\Omega, A, \mu)\right.$, para todo $\left.n \in \mathbb{N}\right)$ la cual converge en casi todas partes a una función medible real-valuadaf. Si existe una función integrable $g$ tal que $\left|f_{n}\right| \leq g$ para todo $n \in \mathbb{N}$, entonces $f$ es integrable $y$

$$
\int f d \mu=\lim _{n \rightarrow+\infty} \int f_{n} d \mu
$$

\section{Referencias}

[1] R. G. Bartle, (1995) The Elements of Integration and Lebesgue Measure, Wiley-Interscience; 1 edition.

[2] D. Moya-Álvarez, (1994) El campo de acción. Una nueva interpretación de la Mecánica Cuántica, editado por la Escuela Politécnica Nacional, Quito, Ecuador.

[3] C. Fefferman, (1983) The uncertainty principle, Bulletin (New Series) of the American Mathematical Society 9, No. 2, $129-206$.

[4] A. Galindo \& P. Pascual, (1990) Quantum Mechanics I, Springer, 1 edition. 